\title{
PSR B2111+46: a test of the inverse Compton scattering model of radio emission
}

\author{
H. Zhang ${ }^{1}$, G. J. Qiao ${ }^{2}$, J. L. Han ${ }^{1}$, K. J. Lee ${ }^{2}$, and H. G. Wang ${ }^{3}$ \\ 1 National Astronomical Observatories, Chinese Academy of Sciences, Jia 20 Datun Road, Beijing 100012, PR China \\ e-mail: [zhanghui;hjl]@bao.ac.cn \\ 2 Department of Astronomy, Peking University, Bejing 100871, PR China \\ e-mail: gin@pku.edu.cn;k.j.lee@water.pku.edu.cn \\ 3 Center for Astrophysics, Guangzhou University, Guangzhou 510400, PR China \\ e-mail: cosmic008@263.net
}

Received 11 October 2006 / Accepted 19 December 2006

\section{ABSTRACT}

\begin{abstract}
The region in which pulsars radiate is very important to constrain pulsar emission mechanisms. A three-dimensional (3D) method to calculate emission heights is developed in this paper. As an example, radiation regions for different components at different frequencies have been calculated for PSR B2111+46. Emission components at seven frequencies are fitted with Gaussian components, then the radiation heights for emission components are calculated. We found that different emission components, even if at the same radio frequency, are radiated from different heights. This is probably a common phenomena and challenges any emission mechanisms. The inverse Compton scattering emission model for pulsar radio emission can explain these results well.
\end{abstract}

Key words. stars: pulsars individual: PSR B2111+46 - radiation mechanisms: non-thermal

\section{Introduction}

Almost forty years have passed since pulsars were discovered However, how pulsars radiate is still an unsolved question. Many radiation models have been suggested for radio emission, such as Ruderman \& Sutherland (1975), Qiao \& Lin (1998), Melrose \& Gedalin (1999), Lyutikov et al. (1999), Gil \& Sendyk (2000), and so on. The determination of where pulsar radio emission is produced is very important to constrain any theories.

The heights of the emission region have been estimated with different methods (Cordes 1978; Blaskiewicz 1991; Phillips 1992; Gil \& Kijak 1993; Kijak \& Gil 1997; Gupta \& Gangadhara 2001; Gupta \& Gangadhara 2003; Dyks et al. 2004; Gangadhara 2005). In this paper we present a three-dimensional method to calculate the emission height. If we have two geometric parameters for a given pulsar with a known period, $P$, namely the inclination angle $\alpha$ between the rotation axis and the magnetic axis, and the view angle $\zeta$ of the line of sight from the rotation axis, then the heights for any emission components at any frequencies can be calculated. The emission components at different frequencies can be separated by Gaussian fitting developed by Wu et al. (1992) and Kramer (1994). We revisit PSR B2111+46 here, separate its components at seven frequencies, and calculate the emission heights at these frequencies.

The structure of this paper is as follows: In Sect. 2 the component separation by Gaussian fitting to the average pulse profiles of PSR B2111+46 is presented. The method to calculate the emission height is developed in Sect. 3, and applied to PSR B2111+46 in Sect. 4 with detailed discussions on the inverse Compoton scattering (ICS) model and the RS (Ruderman \& Sutherlan 1975) model. Conclusions and discussions are summarized in Sect. 5.

\section{Component-separation by Gaussian fitting to profiles of PSR B2111+46}

Usually a pulsar profile has many components, and each of them is assumed to have a Gaussian shape (Wu et al. 1992, 1998; Kramer 1994; Kramer et al. 1994; Kuzimin \& Izvekova 1996; $\mathrm{Xu} \& \mathrm{Wu} 2003)$. This is natual if one consideres the Gaussian shape of subpulses and statistical properties of individual pulses. So the fitting function for a pulse profile can be written as the sum of several individual Gaussians, as follows:

$y=\sum_{i=1}^{n} I_{i} \exp \left[-4 \ln 2\left(\frac{x-p_{i}}{w_{i}}\right)^{2}\right]$

where $I_{i}$ is the amplitude of the $i$ th Gaussian component, $p_{i}$ is the peak position, and $w_{i}$ is the full width at half maximum. These parameters can be determined from the Gaussian fitting by using the numerical method of downhill simplex algorithm (Caceci \& Cacheris 1984). The root mean square (rms) value of the fitting is given by

$\mathrm{rms}=\sqrt{\left[\sum_{j=1}^{N}\left(y_{j}^{\prime}-y_{j}\right)^{2}\right] / N}$.

Here $y_{j}^{\prime}$ is the data point of an observed profile. $N$ is the total number of data points. For a good fit, the residual curve of the on-pulse part should be noiselike, and the rms value should be close to that of the off-pulse part.

The profiles of PSR B2111+46 at seven frequencies, $408 \mathrm{MHz}, 610 \mathrm{MHz}, 925 \mathrm{MHz}, 1.408 \mathrm{GHz}$ and $1.64 \mathrm{GHz}$ (Gould \& Lyne 1998), and $800 \mathrm{MHz}$ and $1.33 \mathrm{GHz}$ (Arzoumanian et al. 1994), were obtained from the European Pulsar Network 
database (Lorimer et al. 1998). We took several steps to fit the profiles:

(1) Separate the on-pulse and off-pulse parts. The on-pulse window is defined for the pulse part that is greater than $3 \sigma$ (the standard deviation) of the off-pulse noise. Here the $\sigma$ is initially calculated from the whole pulse phase data. But later $\sigma$ is repeatedly calculated after the pulse of $>3 \sigma$ is taken out, until the on-pulse part is no longer increasing.

(2) Fit the Gaussian components to the on-pulse part using Eq. (1), and calculate the residual curve.

(3) Compare the residual value to the off-pulse data. If there is any peak larger than about $3 \sigma$, we add another new component, here $\sigma$ is the standard deviation of off-pulse data. Such a procedure is repeated until the rms values of the on-pulse residual curve and off-pulse data are comparable.

The profiles of PSR B2111+46 appear to have three peaks. We used three Gaussian components to fit them. The residuals have much larger $\sigma$ than that of off-pulse, so it is necessary to add components, until five components are involved (see Fig. 1 and parameters in Table 1). That is to say, this pulsar is of the M-type, not of the T-type suggested by Rankin (1993). The positions of these components are coincident with each other at seven frequencies, and these components evolve smoothly with frequency.

In Table $1, p_{1} \sim p_{5}$ are the peaks of the components of pulses, and $w_{1} \sim w_{5}$ and $I_{1} \sim I_{5}$ are full width and intensity of the Gaussian components, respectively. The uncertainties given for these parameters are estimated by the bootstrap method using "dirty" initial inputs, and the final results are converged in the ranges given in the tables. Using the core-double conal model, it is natual to think that $p_{1}, p_{5}$ correspond to outer conal components; $p_{2}, p_{4}$ correspond to inner conal components; and $p_{3}$ corresponds to the core component. The peak-peak separations of outer conal components and inner conal components can be obtained from component locations (i.e., $p_{1}, p_{5}$ or $\left.p_{2}, p_{4}\right)$. The core component is set at a phase of zero $\left(\phi_{0}=0\right)$ in all the seven frequencies. $E_{i} / E_{t}$ is the relative energy for $i$ th conal component to the total energy, and its evolution with frequency is shown in Fig. 2.

\section{Three-dimensional calculations of the pulsar emission height}

The magnetic field of a pulsar is assumed to have a dipole shape. The direction of radiation is tangential to the magnetic field located at an emission point. We assume that the emission beam is symmetric to the plane that contains the rotation axis $\Omega$ and magnetic axis $\mu$ (hereafter the $\Omega-\mu$ plane, see Fig. 3 ). We temporarily use the last open magnetic field line to calculate the emission height, and will also do it for field lines footed at $75 \%$ of the polar caps radius.

In the framework of the core-conal model, as suggested by Rankin (1983) and demonstrated in Fig. 3, $\alpha$ is the magnetic inclination angle and $\zeta$ is the inclination angle of the observer's line of sight to the rotation axis, so that the impact angle $\beta=$ $\zeta-\alpha, \theta_{\mu}$ is the inclination of radiation direction to the magnetic axis, $\psi$ is the angle between the magnetic field plane and the $\Omega-\mu$ plane, and $\phi$ is the azimuth angle around the rotation axis. According to spherical geometry, we can obtain two equations, as follows:

$\cos \theta_{\mu}=\cos \alpha \cos \zeta+\sin \alpha \sin \zeta \cos \phi$, $\cos \zeta=\cos \alpha \cos \theta_{\mu}-\sin \alpha \sin \theta_{\mu} \cos \psi$

In fact, $\alpha$ and $\zeta$ can be estimated from pulsar polarization data, and the half width $\phi$ between peaks of two conal components can be measured from profile data or the results of Gaussian fitting. Then the beam angle $\theta_{\mu}$ can be figured out using Eq. (3). Then we can also get $\cos \psi$ by Eq. (4), i.e., determine the magnetic field line from which the radiation comes. A given magnetic field line in dipole follows

$r=R_{\mathrm{e}} \sin ^{2} \theta$

where $r$ is the polar radius (counted from pulsar center to an emission point, see Fig. 4), $\theta$ is the polar angle of the emission point, and $R_{\mathrm{e}}$ is the maximum radius of this magnetic field line, which is the function of $\psi$ and $\alpha$. If $\theta$ and $R_{\mathrm{e}}$ can be fixed, then one can get $r$, i.e., the emission height. Now let us try to fix $\theta$ and $R_{\mathrm{e}}$ for the last open field lines that we temporary assumed for emission geometry.

First of all, at the tangent point of the field line, the polar angle $\theta$ and the beam angle $\theta_{\mu}$ can be related by (Qiao \& Lin 1998)

$\tan \theta_{\mu}=\frac{3 \sin 2 \theta}{1+3 \cos 2 \theta}$.

From this equation, $\theta$ can be determined when $\theta_{\mu}$ is calculated using Eq. (3).

To calculate $R_{\mathrm{e}}$, we find the projection of $r$ to the equator plane (i.e., the $X-Y$ plane, see Fig. 4) perpendicular to the rotation axis as

$l=R_{\mathrm{e}} \sin ^{2} \theta \sqrt{1-(\cos \alpha \cos \theta-\sin \alpha \sin \theta \cos \psi)^{2}}$.

Obviously, $l$ reaches the maximum value when the polar radius $r$ passes the tangent point $\mathrm{M}$ to the light cylinder. Here $R_{\mathrm{lc}}=c P / 2 \pi$ is the radius of the light cylinder of this pulsar, $c$ is the light velocity, and $P$ is the period of a given pulsar (see Fig. 4). At the point $\mathrm{M}, \mathrm{d} l / \mathrm{d} \theta=0$, one can find

$A \cot ^{3} \theta_{\mathrm{M}}+B \cot ^{2} \theta_{\mathrm{M}}+C \cot \theta_{\mathrm{M}}+D=0$,

where $A=4 \sin ^{2} \alpha, B=5 \sin 2 \alpha \cos \psi, C=4 \sin ^{2} \alpha-$ $6\left(\sin ^{2} \alpha \cos ^{2} \psi-\cos ^{2} \alpha\right)$, and $D=-\sin 2 \alpha \cos \psi$, and $\theta_{\mathrm{M}}$ is the polar angle at point $\mathrm{M}$. So $\cot \theta_{\mathrm{M}}$ can be numerically figured out using Eq. (8). Then $R_{\mathrm{e}}$ is:

$$
R_{\mathrm{e}}=\frac{R_{\mathrm{lc}}\left(1+\cot ^{2} \theta_{\mathrm{M}}\right)^{3 / 2}}{\sqrt{1+\cot ^{2} \theta_{\mathrm{M}}-\left(\cos \alpha \cot \theta_{\mathrm{M}}-\sin \alpha \cos \psi\right)^{2}}} .
$$

Given the values of $R_{\mathrm{e}}$ and $\psi$, a magnetic field line can be uniformly defined, i.e., the magnetic field line for the emission can be ascertained. In summary, if one finds the half width $\phi$ between two peaks from pulse profile, and obtains the inclination angle $\alpha$ and the impact angle $\beta$ from pulsar polarization, then the emission height can be conclusively ascertained by equations above.

\section{Emission height of PSR B2111+46 and the ICS model}

In the last section we developed a geometrical method to calculate the emission height. Now, we apply the method above to PSR B2111+46. The inclination angle $\alpha$ and the impact angle $\beta$ are $9^{\circ}$ and $1.4^{\circ}$ according to Rankin (1993), or $11.6^{\circ}$ and $-1.0^{\circ}$, respectively, according to Gould (1994). We calculate the emission height at both the last open field line and the field line footed 
H. Zhang et al.: PSR B2111+46: a test of the inverse Compton scattering model of radio emission
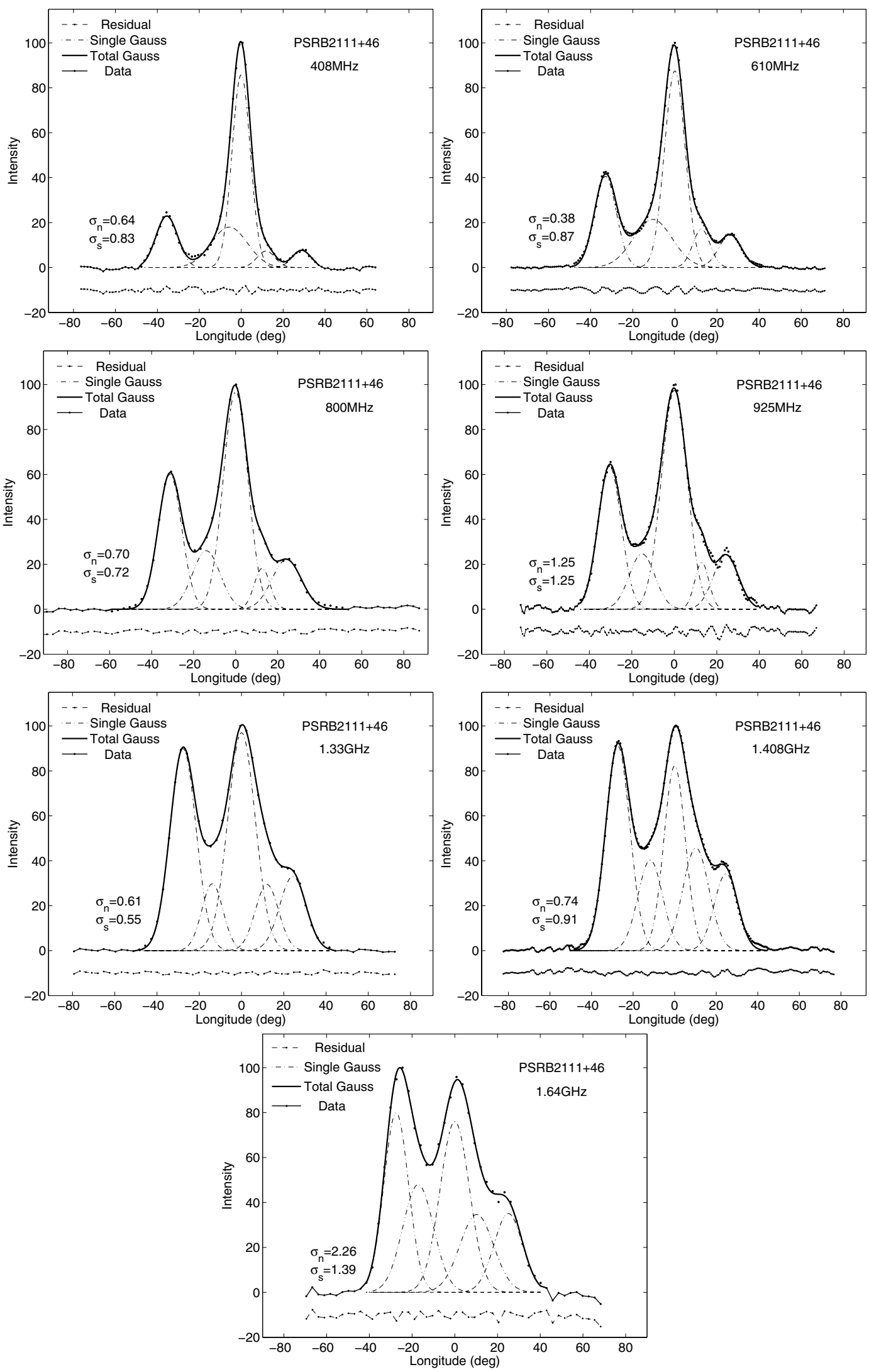

Fig. 1. Gaussian fitting to profiles of PSR B2111+46 at seven frequencies. Data were taken from the European Pulsar Network database (Lorimer et al. 1998). Original profiles at $408 \mathrm{MHz}, 610 \mathrm{MHz}, 925 \mathrm{MHz}, 1.408 \mathrm{GHz}$, and $1.64 \mathrm{GHz}$ were published by Gould \& Lyne (1998), and these at $800 \mathrm{MHz}$ and $1.33 \mathrm{GHz}$ by Arzoumanian et al. (1994). Pulse intensity is in arbitrary units with the peak scaled to 100, and the profiles were centered at the central peak. 
Table 1. Gaussian fitting parameters of different components of PSR B2111+46 at seven frequencies.

\begin{tabular}{rrrrrc}
\hline \hline Frequency & $i$ & \multicolumn{1}{c}{$p_{i}\left({ }^{\circ}\right)$} & \multicolumn{1}{c}{$I_{i}$} & \multicolumn{1}{c}{$\left.w_{i}{ }^{\circ}\right)$} & $E_{i} / E_{t}$ \\
\hline & 1 & $-35.5 \pm 0.1$ & $22.9 \pm 0.1$ & $12.1 \pm 0.1$ & $0.17 \pm 0.01$ \\
& 2 & $-3.1 \pm 4.7$ & $18.5 \pm 4.7$ & $20.9 \pm 3.8$ & $0.23 \pm 0.08$ \\
$408 \mathrm{MHz}$ & 3 & 0 & $82.8 \pm 4.2$ & $10.2 \pm 0.3$ & $0.51 \pm 0.05$ \\
& 4 & $11.2 \pm 4.4$ & $6.5 \pm 4.7$ & $9.6 \pm 3.7$ & $0.04 \pm 0.03$ \\
& 5 & $28.9 \pm 0.1$ & $7.6 \pm 0.2$ & $10.6 \pm 0.7$ & $0.05 \pm 0.01$ \\
\hline & 1 & $-32.9 \pm 0.1$ & $40.3 \pm 0.2$ & $12.3 \pm 0.1$ & $0.21 \pm 0.01$ \\
& 2 & $-7.7 \pm 1.3$ & $24.6 \pm 1.5$ & $24.8 \pm 1.5$ & $0.26 \pm 0.02$ \\
$610 \mathrm{MHz}$ & 3 & 0 & $80.2 \pm 3.3$ & $11.1 \pm 0.2$ & $0.38 \pm 0.02$ \\
& 4 & $12.6 \pm 0.1$ & $14.6 \pm 1.4$ & $9.5 \pm 0.2$ & $0.06 \pm 0.01$ \\
& 5 & $26.2 \pm 0.1$ & $14.4 \pm 0.2$ & $12.8 \pm 0.2$ & $0.08 \pm 0.01$ \\
\hline & 1 & $-31.5 \pm 0.1$ & $59.4 \pm 0.5$ & $13.2 \pm 0.1$ & $0.26 \pm 0.01$ \\
& 2 & $-12.9 \pm 1.1$ & $28.3 \pm 2.0$ & $17.7 \pm 1.9$ & $0.16 \pm 0.02$ \\
$800 \mathrm{MHz}$ & 3 & 0 & $92.1 \pm 4.8$ & $13.3 \pm 0.5$ & $0.40 \pm 0.03$ \\
& 4 & $12.2 \pm 0.3$ & $19.6 \pm 2.1$ & $9.5 \pm 1.1$ & $0.06 \pm 0.01$ \\
& 5 & $24.3 \pm 0.4$ & $22.0 \pm 0.4$ & $16.3 \pm 0.7$ & $0.12 \pm 0.01$ \\
\hline & 1 & $-31.0 \pm 0.2$ & $59.5 \pm 2.5$ & $12.4 \pm 0.3$ & $0.24 \pm 0.02$ \\
& 2 & $-13.6 \pm 1.4$ & $27.6 \pm 1.6$ & $21.9 \pm 4.5$ & $0.20 \pm 0.04$ \\
$925 \mathrm{MHz}$ & 3 & 0 & $88.5 \pm 5.6$ & $13.3 \pm 0.6$ & $0.39 \pm 0.04$ \\
& 4 & $12.8 \pm 0.3$ & $24.3 \pm 1.8$ & $9.4 \pm 0.9$ & $0.08 \pm 0.01$ \\
& 5 & $24.8 \pm 0.2$ & $24.2 \pm 0.4$ & $12.1 \pm 0.4$ & $0.10 \pm 0.01$ \\
\hline & 1 & $-28.8 \pm 0.2$ & $86.5 \pm 2.2$ & $14.1 \pm 0.2$ & $0.30 \pm 0.02$ \\
& 2 & $-13.1 \pm 0.8$ & $37.7 \pm 3.2$ & $16.9 \pm 2.7$ & $0.16 \pm 0.03$ \\
$1.64 \mathrm{GHz}$ & 3 & 0 & $89.3 \pm 5.2$ & $14.1 \pm 0.7$ & $0.31 \pm 0.03$ \\
& 4 & $11.3 \pm 1.3$ & $29.0 \pm 7.9$ & $13.3 \pm 2.6$ & $0.09 \pm 0.03$ \\
& 5 & $23.6 \pm 0.6$ & $38.1 \pm 2.5$ & $15.4 \pm 0.6$ & $0.14 \pm 0.01$ \\
\hline & 3 & 0 & $81.9 \pm 6.9$ & $15.3 \pm 1.3$ & $0.29 \pm 0.04$ \\
& 4 & $11.2 \pm 0.7$ & $31.6 \pm 4.9$ & $12.4 \pm 1.6$ & $0.10 \pm 0.02$ \\
& 5 & $23.4 \pm 0.5$ & $33.5 \pm 1.9$ & $14.8 \pm 0.6$ & $0.12 \pm 0.01$ \\
\hline & 1 & $-28.1 \pm 0.1$ & $91.6 \pm 0.6$ & $13.8 \pm 0.1$ & $0.32 \pm 0.01$ \\
& 2 & $-14.0 \pm 0.6$ & $32.4 \pm 3.3$ & $12.2 \pm 1.2$ & $0.10 \pm 0.01$ \\
& 0 & $98.3 \pm 2.3$ & $15.3 \pm 1.0$ & $0.38 \pm 0.03$ \\
& 4 & $11.7 \pm 0.4$ & $23.9 \pm 5.1$ & $9.1 \pm 1.1$ & $0.06 \pm 0.01$ \\
& 5 & $22.7 \pm 0.3$ & $38.0 \pm 0.7$ & $14.7 \pm 0.3$ & $0.14 \pm 0.01$ \\
\hline & 1 & $-28.4 \pm 0.3$ & $81.5 \pm 6.5$ & $13.9 \pm 0.3$ & $0.26 \pm 0.03$ \\
& & & & \\
\hline & & &
\end{tabular}

at $75 \%$ of the radius of polar cap. The pulsar period is $1.015 \mathrm{~s}$ and its radius is assumed to be $10 \mathrm{~km}$.

With the $3 \mathrm{D}$ method above, the beam angle $\theta_{\mu}$ and radio emission height $r$ can be worked out using Eq. (3)-(9), as listed in Table 2. The emission heights are very low in the pulsar magnetosphere, no less than $1 \%$ of the radius of the light cylinder $\left(R_{\mathrm{lc}}=48462.7 \mathrm{~km}\right)$, The heights calculated with $\alpha, \beta$ of Gould (1994) are larger than those with $\alpha, \beta$ of Rankin (1993). In general, the emission height of the outer conal components is about a few hundred $\mathrm{km}$, but that of the inner conal components is about a few tens of $\mathrm{km}$, and that of the core component is very small, only about ten kilometers above the surface. The emission height for the outer conal decreases when the radiation frequency increases, which is good, in accordance with the "radius-to-frequency mapping" (Ruderman \& Sutherland 1975; Cordes 1978; Phillps 1992). However, this is not the case for the inner cone. The emission height of inner cone increases with frequency. As we will see below, this can be explained by the inverse Compton scattering (ICS) model.

Qiao (1988a,b) first advanced the ICS model. The main idea of this model is as follows: low frequency photons were assumed to be created near the polar cap region by periodic breakdown of the inner gap (Ruderman \& Sutherland 1975). They propagated outwards to the emission region of interest, and were inverse

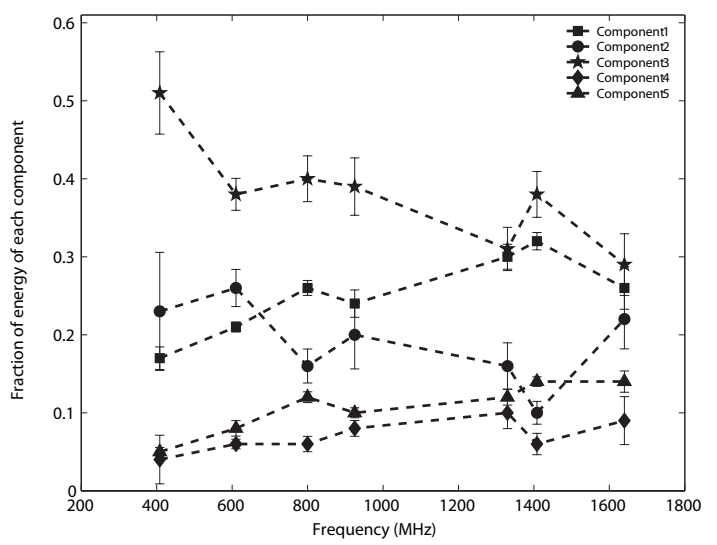

Fig. 2. The frequency evolution of the energy fraction of each component.

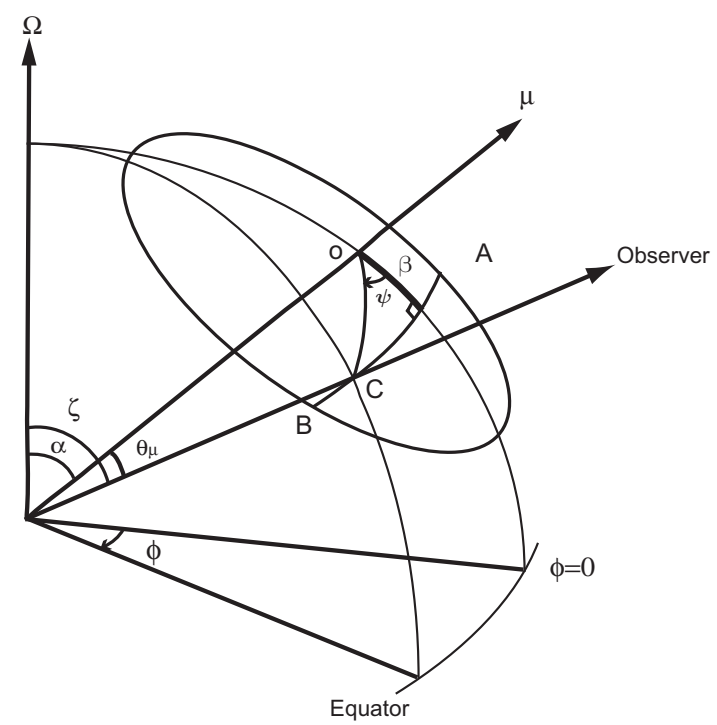

Fig. 3. The geometry of pulsar emission. We define $\phi=0$ at the $\Omega-\mu$ plane. $\mathrm{C}$ is a point that we observed the emission when the line of sight impacts the beam from $\mathrm{A}$ to $\mathrm{B}$.

Compton scattered by the high energy secondary particles that were produced by the gap (see Fig. 5). The up-scattered radio photons, i.e., the observed radio emission are related to the incoming photons by (Xia et al. 1985; Qiao 1988a,b; Qiao \& Lin 1998):

$\omega^{\prime}=2 \gamma^{2} \omega_{0}\left(1-\eta \cos \theta_{i}\right)$,

$\cos \theta_{i}=\frac{M}{N}$

where $M=2 r \cos \theta-R\left[3 \cos \theta \sin \theta \sin \theta_{c} \cos \left(\psi-\psi_{\mathrm{c}}\right)+\left(3 \cos ^{2} \theta-\right.\right.$ 1) $\left.\cos \theta_{\mathrm{c}}\right]$, and $N=\left(1+3 \cos ^{2} \theta\right)^{1 / 2}\left\{r^{2}+R^{2}-2 r R\left[\cos \theta \cos \theta_{\mathrm{c}}+\right.\right.$ $\left.\left.\sin \theta \sin \theta_{\mathrm{c}} \cos \left(\psi-\psi_{\mathrm{c}}\right)\right]\right\}^{1 / 2}$. Here $\theta_{i}$ is the angle between the direction of particle motion and the incoming photon, $\omega_{0}$ is the photon angular frequency, assumed to be $10^{6} \mathrm{~Hz}, \eta=v / c \simeq 1$, $\gamma$ is the Lorentz factor, $\psi$ and $\psi_{\mathrm{c}}$ are the azimuth angle of the magnetic field line and the spark point, and $\theta_{\mathrm{c}} \simeq(2 \pi R / P c)^{1 / 2}$ is the polar cap angle of a last open field line (Qiao \& Lin 1998). The Lorentz factor of particles are assumed to follow (Qiao \& Liu 2001):

$\gamma=\gamma_{0} \exp \left(-\kappa \frac{r-R}{R}\right)$ 
Table 2. Emission heights of different components of PSR B2111+46 at seven frequencies calculated with the 3D geometrical method.

\begin{tabular}{lrcrrrr}
\hline \hline & & \multicolumn{3}{c}{$\alpha=9^{\circ}, \beta=1.4^{\circ}$} & \multicolumn{2}{c}{$\alpha=11.6^{\circ}, \beta=-1^{\circ}$} \\
Frequency & $\Delta \phi\left(^{\circ}\right)$ & $\theta_{\mu}\left({ }^{\circ}\right)$ & \multicolumn{1}{c}{$r(\mathrm{~km})^{a}$} & \multicolumn{1}{c}{$r(\mathrm{~km})^{b}$} & $\left.\theta_{\mu}{ }^{\circ}\right)$ & \multicolumn{1}{c}{$r(\mathrm{~km})^{b}$} \\
\hline \multirow{3}{*}{$408 \mathrm{MHz}$} & $64.4 \pm 0.2$ & $5.5 \pm 0.1$ & $199.9 \pm 0.6$ & $355.0 \pm 1.5$ & $6.2 \pm 0.1$ & $446.6 \pm 2.0$ \\
& $14.3 \pm 6.6$ & $1.9 \pm 0.4$ & $22.3 \pm 6.6$ & $39.7 \pm 11.6$ & $1.7 \pm 0.5$ & $33.7 \pm 15.2$ \\
& 0 & 1.4 & 13 & 23 & 1.0 & 11.7 \\
\hline \multirow{3}{*}{$610 \mathrm{MHz}$} & $20.3 \pm 1.3$ & $2.2 \pm 0.1$ & $31.9 \pm 1.8$ & $56.7 \pm 3.2$ & $2.2 \pm 0.1$ & $56.0 \pm 4.2$ \\
& 0 & 1.4 & 13 & 23 & 1.0 & 11.7 \\
\hline & $55.8 \pm 0.4$ & $4.9 \pm 0.1$ & $153.9 \pm 1.5$ & $274.0 \pm 2.6$ & $5.4 \pm 0.1$ & $340.5 \pm 3.5$ \\
$800 \mathrm{MHz}$ & $25.1 \pm 1.2$ & $2.5 \pm 0.1$ & $41.8 \pm 2.0$ & $74.5 \pm 3.7$ & $2.6 \pm 0.1$ & $79.3 \pm 4.8$ \\
& 0 & 1.4 & 13 & 23 & 1.0 & 11.7 \\
\hline & $55.9 \pm 0.3$ & $4.9 \pm 0.1$ & $154.4 \pm 1.0$ & $274.8 \pm 2.0$ & $5.4 \pm 0.1$ & $341.6 \pm 2.6$ \\
$925 \mathrm{MHz}$ & $26.4 \pm 1.5$ & $2.6 \pm 0.1$ & $45.0 \pm 2.6$ & $80.0 \pm 4.8$ & $2.7 \pm 0.1$ & $86.5 \pm 6.3$ \\
& 0 & 1.4 & 13 & 23 & 1.0 & 11.7 \\
\hline \multirow{3}{*}{$1.33 \mathrm{GHz}$} & $24.3 \pm 1.1$ & $2.5 \pm 0.1$ & $40.2 \pm 1.8$ & $71.3 \pm 3.3$ & $2.5 \pm 0.1$ & $75.1 \pm 4.3$ \\
& 0 & 1.4 & 13 & 23 & 1.0 & 11.7 \\
\hline \multirow{3}{*}{$1.408 \mathrm{GHz}$} & $50.9 \pm 0.3$ & $4.5 \pm 0.1$ & $130.7 \pm 0.9$ & $232.5 \pm 1.8$ & $5.0 \pm 0.1$ & $286.2 \pm 2.4$ \\
& $25.7 \pm 0.7$ & $2.6 \pm 0.1$ & $43.4 \pm 1.2$ & $77.0 \pm 2.2$ & $2.7 \pm 0.1$ & $82.6 \pm 3.3$ \\
& 0 & 1.4 & 13 & 23 & 1.0 & 11.7 \\
\hline \multirow{3}{*}{$1.64 \mathrm{GHz}$} & $52.0 \pm 0.7$ & $4.6 \pm 0.1$ & $135.7 \pm 2.3$ & $241.5 \pm 4.3$ & $5.1 \pm 0.1$ & $298.0 \pm 5.7$ \\
& $27.6 \pm 2.2$ & $2.7 \pm 0.2$ & $48.1 \pm 4.2$ & $85.3 \pm 7.4$ & $2.8 \pm 0.2$ & $93.4 \pm 9.7$ \\
& 0 & 1.4 & 13 & 23 & 1.0 & 11.7 \\
\hline
\end{tabular}

${ }^{a}$ The maximum radius of the last open field line; ${ }^{b} 75 \%$ of the polar cap radius.

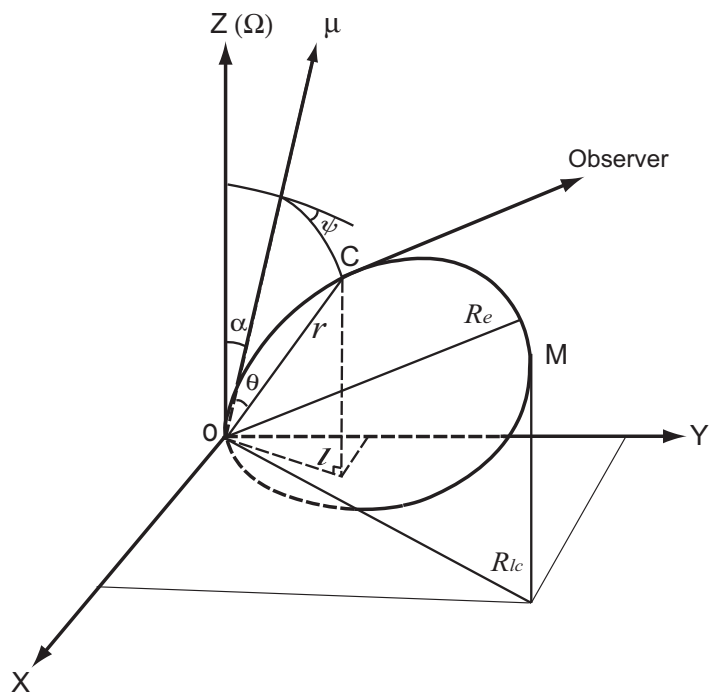

Fig. 4. The geometry of a last open field line. $r$ and $\theta$ are the polar radius and polar angle, respectively. $\mathrm{M}$ is the tangent point of the field line to the light cylinder. $l$ is the projection of polar radius $r$ to the equator plane. $\alpha, \psi$ has the same meaning as in Fig. 3. The radius of the light cylinder is $R_{\mathrm{lc}}$.

where $\gamma_{0}$ is the initial Lorentz factor, and $\kappa$ is a factor for the energy loss of particles. We will also try the linear decay of the Lorentz factor later. The emission frequency and emission height can be figured out in the ICS model by Eqs. (10)-(12). In Fig. 6, the lines are calculated for the last open field line in the $\Omega-\mu$ plane and we use the Lorentz factor above. In Figs. 7 and 8, data points are re-calculated for the field lines footed at $75 \%$ of the polar radius cap in the $\Omega-\mu$ plane, and the ICS model for the emission region calculation had a linear decay of the Lorentz factor as follows:

$\gamma=\gamma_{0}\left(1-\kappa \frac{r-R}{R_{\mathrm{e}}}\right)$,

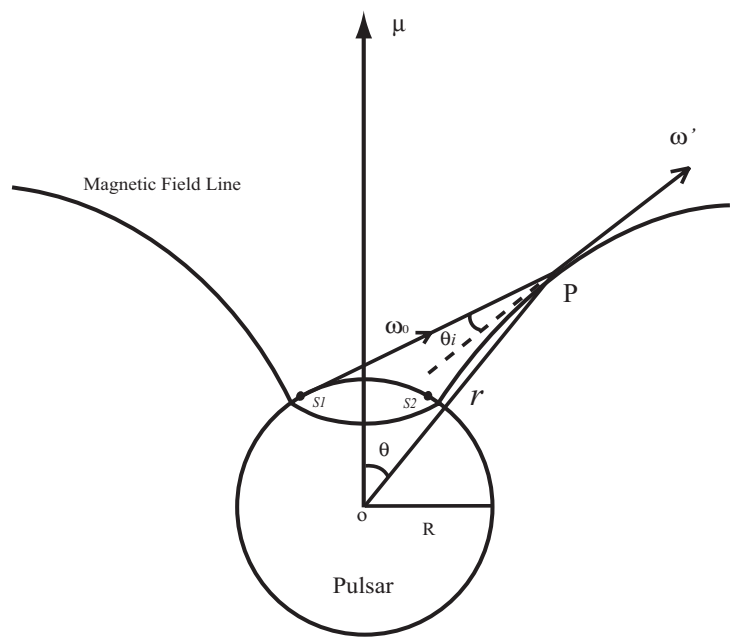

Fig. 5. Geometry for the inverse Compton scattering model. The lowfrequency photons of $\omega_{0}$ produced at sparking point $S 1$ are upscattered by high-energy particles at point $P$ and created the outgoing photons of $\omega^{\prime}$ that we observed as the radio emission. Here, $\theta_{i}$ is the angle between the direction of particle motion, i.e., tangential direction of the magnetic field, and the incoming photon. $S 1$ and $S 2$ are two sparking points on the polar near (but not or) the footpoints of the field lines.

where $\gamma_{0}$ is the initial Lorentz factor, $\kappa$ is a factor for the energy loss of particles, and $R_{\mathrm{e}}$ is the maximum radius of the magnetic field line. The sparking on the polar cap is assumed to produce photons of a few frequency of $10^{6} \mathrm{~Hz}$. Two sparking points ( $\mathrm{S} 1$, $\mathrm{S} 2$ in Fig. 5) from two sides of the magnetic axis are chosen near to (but not or) the footpoints of field lines in the $\Omega-\mu$ plane. Each of them were labeled with three different line styles, which represented emission regions for three components. Other possible sparkings should produce emission between these two curves. It is clear that in the framework of the ICS model, we can explain the relation between the emission height and radiation frequency, especially the abnormal widening of the inner cone with increasing frequency. 

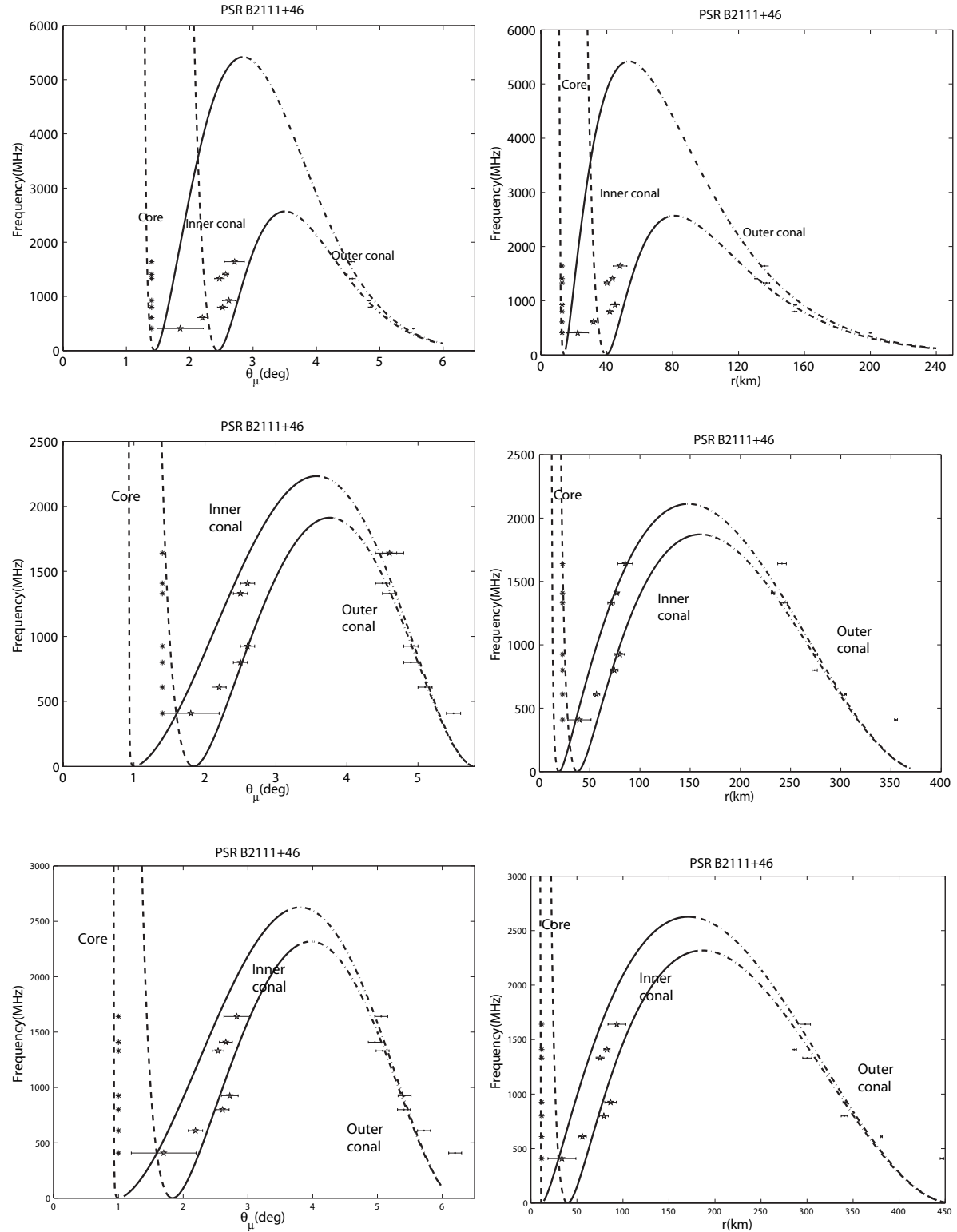

Fig. 6. The data points were calculated at the last open field line with the geometrical method in $\S 3$, by taking $\alpha=9^{\circ}, \beta=1.4^{\circ}$ from Rankin (1993). Two curves were calculated from two sparking points $S 1$ and $S 2$ at 95\% of the polar cap radius in the $\Omega-\mu$ plane. A Lorentz factor decay was taken as $\gamma=\gamma_{0} \exp \left(-\kappa \frac{r-R}{R}\right), \gamma_{0}=30000, \kappa=0.155$. The core component should be between the dash lines, the inner conal component between the solid lines, and the outer cone component between the dash-dot-dash lines.

Fig. 7. The data points were calculated at the field lines footed at $75 \%$ of the polar cap radius by taking $\alpha=9^{\circ}, \beta=1.4^{\circ}$ from Rankin (1993). Two curves were calculated for the two sparking points $S 1$ and $S 2$ at $74 \%$ of the polar cap radius in the $\Omega-\mu$ plane. A linear decay of the Lorentz factor was taken as $\gamma=\gamma_{0}\left(1-\kappa \frac{r-R}{R_{\mathrm{e}}}\right)$, and $\gamma_{0}=10000$ and $\kappa=225$.

Fig. 8. The same as Fig. 7, but the data were calculated with $\alpha=11.6^{\circ}, \beta=-1.0^{\circ}$ from Gould (1994) and the ICS model with $\gamma_{0}=$ $10000, k=195$.
The Lorentz factors we used for fitting are 30000 and 10000 , larger than those of given by the RS model, which set the Lorentz factor for the secondary particles as about 800 (Ruderman \& Sutherland 1975). From both the theoretical and observational points of view, the results we obtained are reasonable. Theoretically, in the RS model, the Lorentz factors of primary particles are $10^{6}$. This value is required from the pair production, and assumed for all primary particles. In fact, not all pairs were produced at the bottom of the gap, and the primary particles cannot gain such a large Lorentz factor. Therefore, particles following from the polar gap, including the secondary particles, should have a Lorentz factor lower than $10^{6}$. Ruderman $\&$ Sutherland (1975) took a Lorentz factor of secondary particles as 800 to produce radio emission by the curvature radiation mechanism. If the sparking in the gap is dominated by the ICS process, then the Lorentz factor of the secondary would be $3.5 \times 10^{3}$ (Zhang et al. 1997). If some pairs are produced at the bottom of the gap, the Lorentz factor could be larger, up to the values we are using for the modeling. Anyway, the range for Lorentz factors are model-dependent at present. From the observational point of view, our fitting results are also reasonable. The microstructures of a sub-pulse often are only a few microseconds wide (Bartel \& Hankins 1982; Hankins et al. 2003). If this comes from particle emission, it should mean that the Lorentz factor of particles should be about $10^{4}$, and that the emission region should be about a kilometer (in size).

Energy losses due to the ICS process on thermal surface $\mathrm{X}$-rays can be rapid, but are extremely sensitive to the particle Lorentz factor in the resonant scattering regime. For the thermal resonant ICS gap, the Lorentz factor of the primary is about $10^{5}$, and in this case, $-\dot{\gamma} \propto B(z)^{2} / \gamma$ (Zhang et al. 1997); here $z$ is the height above the surface. This means that the energy loss is very fast. In the non-resonant region, $-\dot{\gamma} \propto \gamma^{4} / B(z)^{2}$ (Zhang et al. 1997). In our case the Lorentz factor reaches $10^{4}$; it is not so far from the resonant point. According to the calculation, when the Lorentz factor is as large as this, the difference of energy loss is not so big (see Xia et al. 1985). We did not make an accurate calculation for $-\dot{\gamma}$ in this paper. It can be a parameter adjusted by the observations, and can constrain the theoretical parameters.

\section{Conclusions and discussions}

We separate the components of PSR B2111+46 at seven frequencies by using Gaussian fitting. Strong evidence shows that 
pulsar profiles have five components, evolving with frequency. Using the peaks separation of these components, the radio emission regions in the pulsar magnetosphere for seven frequencies have been found by assuming dipole structure of magnetic field. Our geometrical calculations were made for the last open field line and the field line footed at $75 \%$ of the polar cap radius. If the field line is closer to the center of the polar cap, the emission height should be even higher than our results. In our calculation, the separation of two outer-conal components or the two inner-conal components, i.e., the open angle of the conal beam, have been used. The retardation and aberration are related to the displacements between the core and the middle-point of conal pairs (see Xu et al. 1997), which are not important for our calculation.

For the outer conal beam, the emission frequency decreases when the emission height increases. For the inner conal beam, in contrast, the emission frequency increases with height. The inner cone emission should come from a region closer to the neutron star surface. The radio emission of different components at a given frequency can be emitted from different heights. These can be easily explained by the ICS model.

Acknowledgements. We are grateful to the anonymous referee for the helpful comments and suggestions. We also appreciate particular valuable discussions with X. J. Wu. Authors are supported by the National Natural Science Foundation of China (Nos. 10521001, 10473015, 10373002, and 10403001).

\section{References}

Arzoumanian, Z., Nice, D. J., Taylor, J. H., \& Thorsett, S. E. 1994, ApJ, 422, 671

Bartel, N., \& Hankins, T. H. 1982, ApJ, 254, L35

Blaskiewicz, M., Cordes, J. M., \& Wasserman, I. 1991, ApJ, 370, 643
Caceci, M. S., \& Cacheris, W. P. 1984, Byte, 9, 340

Cordes, J. M. 1978, ApJ, 222, 1006

Dyks, J., Rudak, B., \& Harding, A. K. 2004, ApJ, 607, 939

Gangadhara, R. T. 2005, ApJ, 628, 923

Gangadhara, R. T., \& Gupta, Y. 2001, ApJ, 555, 31

Gil, J. A., \& Kijak, J. 1993, A\&A, 273, 563

Gil, J. A., \& Sendyk, M. 2000, ApJ, 541, 351

Gould, D. M. 1994, Ph.D. Thesis, University of Manchester

Gould, D. M., \& Lyne, A. G. 1998, MNRAS, 301, 235

Gupta, Y., \& Gangadhara, R. T. 2003, ApJ, 157, 869

Hankins, T. H., Kern, J. S., Weatherall, J. C., \& Eilek, J. A. 2003, Nat 422, 141

Kijak, J., \& Gil, J. 1997, MNRAS, 288, 631

Kramer, M. 1994, A\&AS, 107, 527

Kramer, M., Wielebinski, R., Jessner, A., Gil, J. A., \& Seiradakis, J. H. 1994, A\&AS, 107, 515

Kuzmin, A. D., \& Izvekova, V. A. 1996, Astron. Lett., 22, 394

Lorimer, D. R., Jessner, A., Seiradakis, J. H., et al. 1998, A\&AS, 128, 541

Lyne, A. G., \& Manchester, R. N. 1988, MNRAS, 234, 477

Lyutikov, M., Blandford, R. D., \& Machabeli, F. 1999, MNRAS, 305, 338

Melrose, D. B., \& Gedalin, M. E. 1999, ApJ, 521, 351

Phillips, J. A. 1992, ApJ, 385, 282

Qiao, G. J. 1988a, Vist. Astron., 31, 393

Qiao, G. J. 1988b, in: High Energy Astrophysics, ed. G. Borner (New York: Springer-Verag), 88

Qiao, G. J., \& Lin, W. P. 1998, A\&A, 333, 172

Qiao, G. J. Liu, J. F. Zhang, B., \& Han, J. L. 2001, A\&A, 377, 964

Rankin, J. M. 1983, ApJ, 274, 333

Rankin, J. M. 1993, ApJS, 85, 145

Ruderman, M. A., \& Sutherland P. G. 1975, ApJ, 196, 51

Wu, X. J., Xu, W., \& Rankin, J. M. 1992, in The Magnetospheric Structure and Emission Mechanisms of Radio Pulsars, ed. T. H. Hankins, J. M. Rankin, \& J. A. Gil (Zielona Góra, Poland: Pedagogical Univ. Press), IAU Coll., 128, 172

Wu, X. J., Gao, X. Y., Rankin, J. M., \& Malofeev, V. M. 1998, ApJ, 116,1984

Xia, X. Y., Qiao, G. J., Wu, X. J., \& Hou, Y. Q. 1985, A\&A, 152, 93

Xu, R. X., Qiao, G. J., \& Han, J. L. 1997, A\&A, 323, 395

Xu, X. B., Wu, X. J. 2003, Science in China, Series G, 46, 104

Zhang, B., Qiao, G. J., \& Han, J. L. 1997, ApJ, 491, 891 